\title{
Bleomycin/Dexamethasone/Lomustine/Vinblastine Regimen
}

National Cancer Institute

Source

National Cancer Institute. Bleomycin/Dexamethasone/Lomustine/Vinblastine Regimen.

NCl Thesaurus. Code C161955.

A chemotherapy regimen consisting of bleomycin, dexamethasone, lomustine, and vinblastine that may be used in the treatment of Hodgkin lymphoma (HL). 